\title{
Absolute Prioritization of Planetary Protection, Ethics, and Avoiding Imperialism in All Future Science Missions: A Policy Perspective
}

Authors: Monica Vidaurri (Howard University, NASA GSFC, monica.r.vidaurri@nasa.gov), Alia Wofford (Howard University, NASA GSFC), Jonathan Brande (University of Kansas), Gabriel Black-Planas (Virginia Tech), Shawn Domagal-Goldman (NASA GSFC)

Co-Signers:

Sarah E. Moran, Johns Hopkins University

Shirin Zaidi, Unaffiliated

Jake Strang, JHU/APL

D.J. Teal, University of Maryland College Park

Michaela Leung, University of California Riverside

Sofia Sheikh, Pennsylvania State University

Briley Lewis, UCLA

Amit Kahana, The Weizmann Institute of Science

Daria Pidhorodetska, University of California Riverside

Edward Schwieterman, University of California Riverside

Giannina Guzman Caloca, University of Maryland College Park

Jordan McKaig, Georgia Institute of Technology

Irena Li, NASA JPL

Hector Delgado Diaz, University of Washington

Jaime S. Crouse, SURA/NASA GSFC

Lena Vincent, University of Wisconsin-Madison

Beck E. Strauss, NIST \& NASA GSFC

Brenna Robertson, University of Connecticut

Dany Waller, Johns Hopkins University

Meredith Durbin, University of Washington

Jennifer (JA) Grier, Planetary Science Institute

Sarah Graber, Columbia University

Sarah E. Swiersz, University of Central Florida

Andrew Rivkin, JHU/APL

Sarah Ballard, University of Florida

Eleni Ravanis, European Space Agency/University of Hawai'i at Mānoa

Stephanie Mueller, DePaul University

Gabrielle Engelmann-Suissa, University of Washington

Annie Drechsler, Unaffiliated

Maggie Thompson, University of California Santa Cruz

James Shults, Unaffiliated 
Valeria Urrutia Hurtado, Fisk University

Richard Dawson

Emily Zhang, Columbia University

Eric Lopez, NASA GSFC

Joseph A. Guidry, University of Texas at Austin

Zafar Rustamkulov, Johns Hopkins University

Yasmeen Asali, Yale University

Daniella Scalice, NASA Ames

This is a white paper that will be submitted to the Astro2020 and Planetary Decadal Surveys. $* * * * * * * * * * * * * * * * * * * * * * * * * * *$

The views expressed in this paper do not necessarily represent those of the authors and endorsers or their affiliations. 


\section{Motivation}

A new age in space exploration will also signal a new age of humanity. Our motives for exploring celestial bodies and potentially settling them, for establishing global satellite networks and private services in space, and for searching for life beyond our world cannot be separated from the inherent ethical and societal impacts that they present. Therefore, it is necessary to ensure that we do not repeat humanity's violent and exploitative history in a new frontier that has the capability of rewriting this unfavorable past and serving as a new internationally collaborative and peaceful endeavor that works on behalf of all of humanity, and not just a few well-funded special interests. The changes needed to prioritize ethics, safety, anti-imperialism, and the socioeconomic impacts of space exploration can begin with planetary science. By actively creating these norms from the individual level, the proper chain of command via the respective government can ensure enforcement of standards in the form of strengthened policy, individuals practicing these norms, and broadened goals of a planetary protection office (PPO). In conjunction, collaboration between PPOs and the planetary sciences is equally important in improving microbial detection and decontamination technologies.

\section{Strengthening the Framework - Areas of Consideration}

Universalization of planetary protection, safety, and ethical standards is crucial for all future space exploration directives and missions, but has a complicated and non-static solution. In this case, universalization of standards is heavily reliant upon building and maintaining a regulatory and communicative infrastructure at every level; one that must continuously facilitate discussion of these standards and update accordingly. Delegated roles, carried out by personnel or offices, must be assigned vertically to provide adequate checks and continuous risk assessment starting at mission conception and throughout the lifetime of the mission. These roles will work to build bridges amongst stakeholders in a mission such that all improved standards and norms with respect to ethics, planetary protection, safety, communication, and collaboration are acted upon. Without the implementation of practiced norms from mission management, any formal establishment of law, from the agency level to international level, will not have a solid practical foundation. Consequently, laws will be established with no prior background of how these norms can be improved and how they help.

\section{Bridging the Regulatory Gap and the Role of the Space Agency}

The Outer Space Treaty (OST) places the responsibility of understanding and adhering to exploration safety and bioethical standards for non-government entities on the respective government(s) and relative agencies representing the entity., ${ }^{3,4}$ Therefore, actions must be taken by governments ultimately responsible for these exploratory actions, public and private, to create and enforce a framework that will continuously monitor, communicate, 
and ensure safety and protectionary regulations while upholding the creation of norms to prioritize ethics. This framework need not be an effort solely put forth by a government, but rather a collaborative and cooperative dialogue prioritizing ethics and necessary regulation between all stakeholders in outer space; local communities, academia, government, private sector, etc.

Section 2.2.3. of NPR8020.12D requires non-NASA entities to submit future protectionary procedural outlines to the NASA Planetary Protection Office. For this reason, it would benefit every agency to have a communicative and transparent PPO (as a function of the agency) or equivalent entity to follow and modify these procedures as needed over the course of a mission, while remaining compliant with and communicating the concerns of ethical committees and liaisons.

Furthermore, Section 2.4 of NPR8020.12D states that, at the request of the PPO, mission management shall make arrangements to allow PPO representatives to be present during transport, decontamination, hardware and environment assessments, and writing of documentation relative to safety and protection. ${ }^{4}$ To strengthen this with regard to the proposed framework, the PPO and any personnel or offices responsible for ethical and sociopolitical implications of a mission should be present through the duration of the mission, including mission categorization, with the PPO and ethical advisors scheduling regular assessments of decontamination procedure, adherence to standards, hardware, documentation, communication, ethical procedure, and any other mission detail falling under the norms to be strengthened regarding the topics discussed in this paper.

\section{Reactive to Proactive Safety Procedures and their Jurisdiction}

By establishing and making clear the ethical norms created at mission conception by personnel and a broadened set of PPO goals, space policy can shift to an increasingly proactive nature. In conjunction with a PPO, any existing ethical and safety committees or liaisons sharing this authority and necessary responsibilities throughout the mission may take more active roles. This includes advising and assessing implications a mission poses to the future of planetary protection, ethics, safety, and the larger sociopolitical global landscape, while reporting these implications to the representative government, ushering in a more robust risk-assessment covering scientific, ethical, sociological, and political risks for space missions. The applicability, combined with language changes enforcing the role of the PPO and relevant committees/liaisons as objective and mandatory in all exploration missions - agency/government and non-agency - will ensure that these roles remain of the utmost importance. 
The need for strengthened operations and policies need not be a fully original endeavor. Among others, NASA Policy Directive (NPD) 8020.7G, which accounts for contamination of in- and outbound spacecraft, would act as an adequate procedural foundation on an international scale, and succeed in adopting the aforementioned executable language and delegation of authority while remaining useful in a legal context. Reinstatement to include, for example, quarantine and contamination control (along with the necessary research for this) for crewed missions would also prove useful in this sense as the space science community progresses further into human exploration.

\section{Anti-Imperialism}

"Seeking to prevent a new form of colonial competition" was the very premise to the OST.3 It is critical that international law adopts a custom of anti-imperialism, similar to militarization, with the presentation of these customs from a majority of each state and agency participating in space exploration, as well as the establishment of explicit anti-imperialism as a clause of the OST. ${ }^{7}$ To achieve this, it is important for space-faring nations to remember that colonization and imperialism is a structure; an institutionalized virtue stemming from exploitation of (often native) populations to preserve a central selfish ideal of preserving the way of life as seen by the colonizing nation. In addition, states party to and responsible for elements of scientific outposts or equipment on other worlds should uphold respective jurisdiction over their contributions, utilisation, and liability rights of facilities, equipment, technologies, personnel, and territories.

It is paramount that the astro community not only recognize colonialism and imperialism in the premise of settling other planets/celestial bodies and their orbits for personal gain, but also work together to prevent further colonialism on Earth. A proper check to colonialism from the astro community takes the example of the Thirty Meter Telescope (TMT). ${ }^{8}$ Management of the current set of telescopes in Mauna Kea have established the norms of paying rent, creating a work pipeline for the Native people, creating education outreach programs for Native youth, and actively facilitating communication with Native tribes and businesses, thus gaining the trust and approval of the Native people to build all current telescope operations. However, the continued controversy around Mauna Kea and the TMT should serve as a new regulatory area of consideration: one that includes "no further development, creat[ing] a community-based management authority including environmentalists, native Hawaiians, regulatory agencies, and collect[ing] rent."' Ground-based observing, as well as other astro-science related developments, cannot and should not take precedence over lands belonging to Native populations, or areas where telescope development is potentially harmful towards native ecosystems and the environment. 
Thus, it is imperative that anti-imperialist standards and methods of thinking begin in the early stages of mission concepcion, along with safety and ethics, and from the PI/individual level through the agency/global level, which can be facilitated and held accountable by the creation/utilization of ethics committees and liaisons.

\section{Ensuring the Framework - Norms and Strategies}

Lobbying

In order to preserve and uphold a more future-oriented, ethical, and comprehensive interactive legal and collaborative framework between the public and private sectors and international partners, it would benefit governments to not allow lobbying efforts to streamline certifications, permits, and renewals of legal documentation allowing access to LEO and other celestial bodies/orbits to pass. Though transparency of technologies used by the private sector is critical, it is not needed with the correct framework and agency technology, planetary protection, and ethics officers maintaining constant communication and regular assessments. In addition, private sector advisory committees or delegates (permanent or ad hoc/per mission) within an agency would greatly benefit the ability to properly assess status of projects, needs, recommendations from the private entity. Committees and delegates may also serve to advise on potential legal protectionary standards, and to uphold high standards of transparent communication between agency, government, international partners, and private entity.

The potential for the private sector to contribute to ground-breaking planetary science and space exploration is limitless and exciting. However, gone unchecked and without proper communication and ethical standards of operation, the private sector can prove to be a venue for continued imperialism, inviting actors who may possess exploitative motives that do not work on behalf of all humanity, resulting in space habitation and governance that will embody the ethos of said actors, and only the ethos of said actors.

\section{$\underline{\text { Res Comunis }}$}

The language used by governments and their respective agencies regarding a state presence in space must also reflect true res comunis law. Rather than protecting state interests in space, space is to be protected by all states in collaboration for peaceful exploration and scientific advancement; a true global commons. However, states must also be ready to exercise the full extent of their jurisdiction on their space items, including debris and debris cleanup technologies and methods. In this light, states and their agencies must also adopt a more strict practice of mission review to adhere to the assessment of a mission launch, eventual mission end, and other potentially impactful space activities to the global uses of space. This takes the example of requesting consultation by other international space agencies, or ethical committees/liaisons. 
International Custom and Norms

Like most jurisdiction reached by international courts on the matters of high seas, terra nullius and res comunis, and resource use, the laws created regarding these matters are largely based off of established norms and practices from individuals (PIs), entities (agencies), and governments, including bilateral treaties and agreements. Establishing clear good faith relations, both in practice and in treaty, will set the international framework needed to create strict and preventative protectionary, ethical, and anti-imperialism methods for all future science missions. In this light, all levels of every science mission are encouraged to actively participate in effectively creating the norm of checks and balances so as to not abuse the presence of different entities, both private and public, in space. These checks may include but are not limited to: voluntarily establishing regular mandatory assessments by PPOs throughout the entirety of a mission starting from concept as well as regular mandatory assessments from international law specialists, clear and transparent international/industry partner lines of communication and delegation of authority if applicable, establishing a clear chain of command and representatives from agencies working with private industries to ensure and enforce progressive safety procedures on the private entity, strict committee/delegate presence within the private industry to communicate needs to and from the agency, and any other behavioral suggestions laid out in this paper.

\section{Utilizing the Goals of the Astro-Sciences in Collaboration}

There are countless proposed and in-development instruments and missions intended to challenge our understanding of other worlds, their environments, and how life may exist on other worlds. The ethical, planetary protection, and collaborative strategy for these missions would benefit from committees made up of representatives from all potential mission stakeholders: planetary protection officers, mission astrobiologists, scientific investigators, students, and private sector engineers and executives. For example, astrobiologists can work together with planetary protection officers and vice versa to improve biomatter detection standards. Ethics liaisons and the private sector can work together to create frameworks such that the private sector can continue innovating in an ethical direction, and have a voice in regulatory conversations.

However, such collaboration between members of the space science community, policy community, and the private sector aren't limited to future planetary missions. Current commercial activity could also benefit from interdisciplinary advisory and regulatory action. The recent crash of the Beresheet lunar lander, of which an unreported payload of human DNA and tardigrades were present, posed a new and serious concern to the planetary science community. Though the survivability of the tardigrades and the actual impact to the lunar environment is negligible, the lack of oversight and allowance of an undisclosed 
payload can empower other initiatives to do the same, and may actually impact the environments of the worlds we explore. Cooperative bodies consisting of representatives from government agencies, academic institutions, and commercial interests can be empowered to minimize detrimental impacts on scientific activity and the integrity of other ground- and space-based environments, and maximize innovation from the private sector in compliance with government standards and proactive communication between the public and private sectors. These initiatives and technologies put forth by the private industry with global needs in mind, though they have the ability to benefit communities worldwide, must also recognize that in the mind of one private entity, "global good" may not be an inherent "good" in the eyes of all communities. Thus, global efforts require global input, which can be accomplished by robust communications with all stakeholders. With these concerns in mind, we reiterate that commercial and scientific activity must avoid harm to both terrestrial and space environments, with particular emphasis on maintaining pristine environments and Native lands. ${ }^{10}$

\section{The Suggested Framework}

Suggestions laid out in this paper regarding ethical implications, safety assurances, contamination control and planetary protection, communication, and accountability standards would ideally fall within the earliest stages of the science traceability matrix. Continued and mandatory assessments of these standards throughout the mission must also be coupled with early traceability to ensure that these ideals remain throughout the entire mission timeline, and throughout all aspects of the mission. This way, a consistent watch on ethics, communication, planetary protection, and other policy and social concerns via committees and liaisons catches any possible infringements and potential to set new, improved norms, and pushes the international communities to adopt more updated and proactive standards. Running parallel to internal and transparent monitoring of ethics and communication is the internationally communicative component, capable of acting as a "check" in international relations, and turning reactive international policy into proactive and constantly-updated policies that can be used by a variety of agencies and nations. Though this paper uses the example of NASA and the United States Government, the framework of transparent and proactive standards that are open to critique and custom-setting can be applied to all governments, institutions, and missions.

\section{References.}

[1] National Academies of Sciences E. Review and Assessment of Planetary Protection Policy Development Processes.; 2018. doi:10.17226/25172

[2] Office of Safety and Mission Assurance. Biological Contamination Control for Outbound and Inbound Planetary Spacecraft (NPD 8020.7G). NASA Policy Directive February 19, 1999. 
[3] United Nations Office for Outer Space Affairs. Treaty on Principles Governing the Activities of States in the Exploration and Use of Outer Space, including the Moon and Other Celestial Bodies. The United Nations December 19, 1966.

[4] Office of Safety and Mission Assurance. Planetary Protection Provisions for Robotic Extraterrestrial Missions (NPD 8020.12D). NASA Policy Directive April 20, 2011.

[5] Journal of the British Interplanetary Society, Volume 67, No 1, pp 1-43.

[6] Vakoch, D. A. (2016). In defence of METI. Nature Physics, 12(10), 890.

[7] United Nations Office for Outer Space Affairs. Convention on Registration of Objects Launched into Outer Space (3235 XXIX) Resolution adopted by the General Assembly. The United Nations November 12, 1974.

[8] Knapp, Alex. "Understanding The Thirty Meter Telescope Controversy." Forbes, Forbes Magazine, 12 June 2015.

[9] "Legal Process - Thirty Meter Telescope." Mauna Kea and TMT, Thirty Meter Telescope.

[10] United Nations Educational, Scientific, and Cultural Organization. "Records of the General Conference, 33rd Session, Paris 3-21 October 2005 V.1: Resolutions .” Universal Declaration on Bioethics and Human Rights, Article 18 